\title{
Plasmacytoma to the Axis Mimicking Metastatic Paraganglioma: Circumferential Reconstruction via Posterior Approach
}

\author{
Young-Seop Park, Seung-Jae Hyun, Ki-Jeong Kim, Tae-Ahn Jahng \\ Department of Neurosurgery, Spine Center, Seoul National University Bundang Hospital, Seoul National University \\ College of Medicine, Seongnam, Gyeonggi, Korea
}

Plasmacytoma is a malignant plasma cell tumor growing within soft tissue or the axial skeleton. Here, we present the case of a patient with plasmacytoma of the axis vertebra who underwent decompressive surgery with reconstruction via a posterior approach. The patient was referred because of quadriparesis with severe neck pain. Magnetic resonance imaging revealed a relatively demarcated, highly enhanced mass lesion in a destructed axis, with spinal cord compression. Computed tomography revealed a $5.6 \times 4.3 \mathrm{~cm}$ adrenal mass at the left retroperitoneal space. We suspected the axis lesion to be a metastatic paraganglioma from the adrenal mass. The patient underwent total excision of the tumor under an operative microscope with occipitocervical fixation. Histopathologically, the tumor was shown to be a plasmacytoma. Following the operation, the patient recovered without significant complications. This was a rare case of plasmacytoma in the axis, mimicking metastatic paraganglioma.

Key Words: Plasmacytoma $\cdot$ Axis $\cdot$ Paraganglioma $\cdot$ Posterior surgery $\cdot$ Fusion

\section{INTRODUCTION}

Plasmacytoma is a clonal proliferative disorder of neoplastic plasma cells and is biologically malignant ${ }^{4}$. Plasmacytoma is rare that responsible for only $5 \%$ of plasma cell neoplasms. Plasmacytoma is further classified into solitary plasmacytoma of bone (SPB), extramedullary plasmacytoma (EP), and multiple solitary plasmacytomas that are either primary or recurrent. Among the three, SPB is the most common and occurs as lytic lesions within the axial skeleton ${ }^{3)}$. EP mostly occurs in the upper respiratory tract (85\%), as do the multiple solitary plasmacytomas. In the latter case, either multiple solitary bone or soft tissue lesions must also be present. In almost all cases, radiotherapy is the main choice for curative treatment because plasmacytomas are radiosensitive. However, in some cases, like with this patient, surgical intervention is required. We have discovered that out of the multiple vertebrae affected

- Received: September 19, 2015 - Revised: November 9, 2015

- Accepted: November 10, 2015

Corresponding Author: Seung-Jae Hyun, MD, PhD

Department of Neurosurgery, Seoul National University Bundang Hospital, Seoul National University College of Medicine, 82, Gumi-ro 173beon-gil, Bundang-gu, Seongnam-si, Gyeonggi-do 13620, Korea

Tel: +82-31-787-7164, Fax: +82-31-787-4059

E-mail: hyunsj@snu.ac.kr

凤This is an Open Access article distributed under the terms of the Creative Commons Attribution Non-Commercial License (http://creativecommons.org/ licenses/by-nc/3.0/) which permits unrestricted non-commercial use, distribution, and reproduction in any medium, provided the original work is properly cited. by plasmacytoma, only the axis presented a symptomatic compressive myelopathy. Here, we describe this case of plasmacytoma at the axis, which was treated by decompressive tumor removal and reconstruction via a posterior approach.

\section{CASE REPORT}

A 72-year-old man was admitted to our institute complaining of severe neck pain and quadriparesis without any significant history of trauma. Plain radiographs showed atlantoaxial instability with osteolytic lesions on the C2 (Fig. 1). Magnetic resonance imaging (MRI) showed a well-enhanced soft tissue mass $(5.9 \times 5.5 \times 5.8 \mathrm{~cm})$ involving the whole compartment of $\mathrm{C} 2$ with cortical destruction. The epidural mass extended from the $\mathrm{C} 1$ to $\mathrm{C} 4$ vertebra at the ventral portion of the dura and from the $\mathrm{C} 2$ to $\mathrm{C} 3$ vertebra at the posterior portion (Fig. 2). The spinal cord was compressed at the $\mathrm{C} 2$ vertebra by the mass and showed slightly increased T2 signal change, suggesting compressive myelopathy. The bilateral vertebral arteries were involved at the $\mathrm{C} 2$ level, but retained intact distal flow. Computed tomography (CT) revealed a $5.6 \times 4.3 \mathrm{~cm}$ adrenal mass at the left retroperitoneal space (Fig. 3). Subsequently, the patient underwent an F-18 FDG whole body PET/CT examination, which revealed a hypermetabolic soft tissue mass (SUVmax=5.4) involving the C2 body, lamina, and spinous process. The adrenal mass was mildly hypermetabolic (SUVmax $=2.8$ ), which ordinarily suggests a benign tumor; 


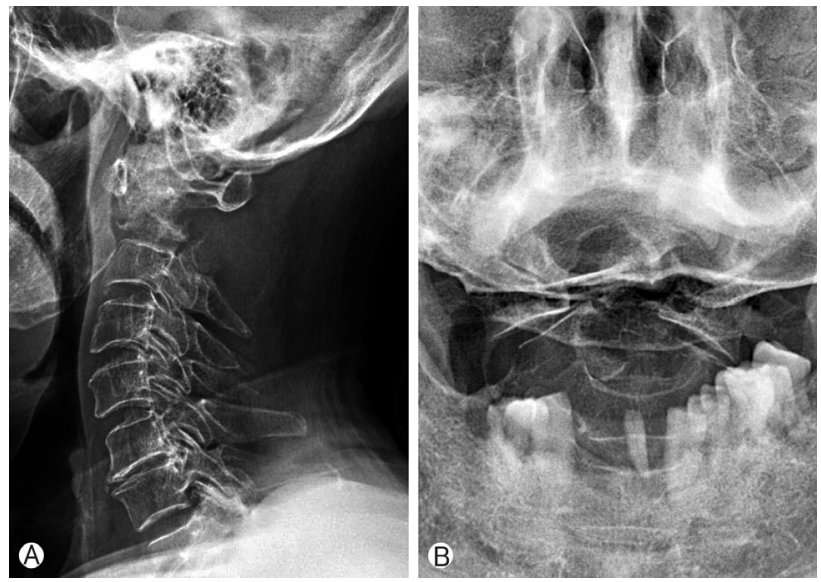

Fig. 1. Plain radiograph showing osteolysis of the axis with malaligment. (A) A lateral cenvical plain radiograph, (B) An open mouth radiograph.
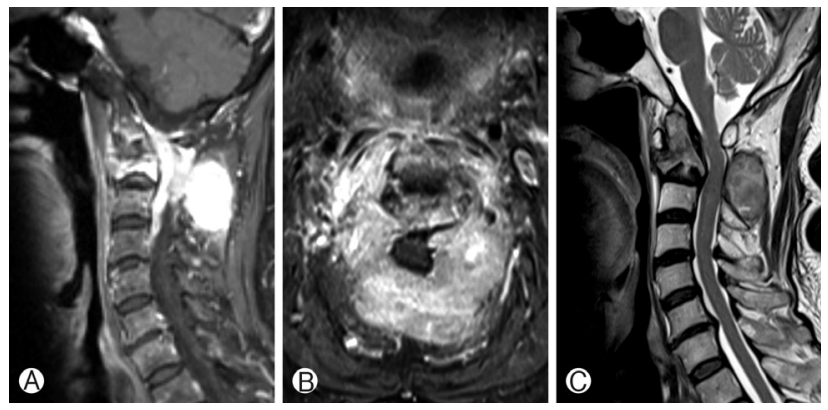

Fig. 2. A mass lesion extending along the anterior epidural space with cord compression at preoperative MRI. (A) Contrast-enhanced sagittal MRI, (B) Contrast-enhanced axial MRI, (C) T2 sagittal MRI.

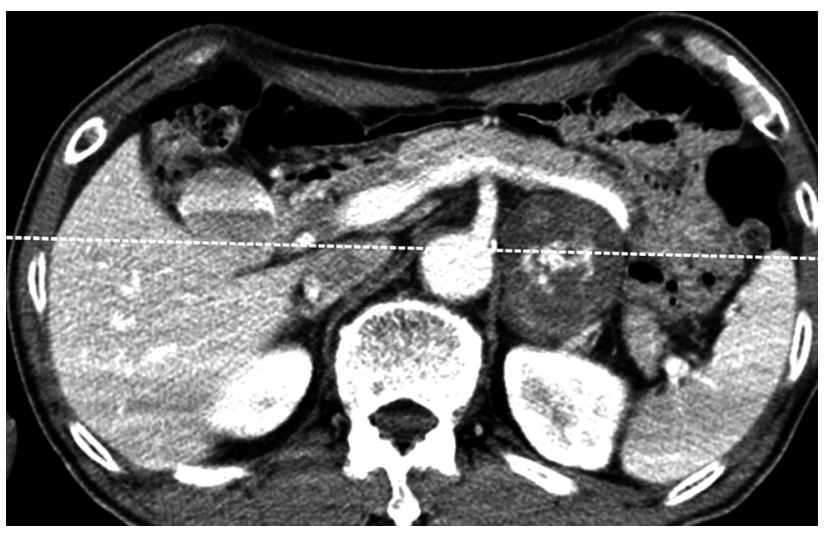

Fig. 3. An abdominal PET/CT image showing a $5.6 \times 4.3 \mathrm{~cm}$ size mass at the left retroperitoneum.

however, the possibility of malignancy remains.

At first, we suspected the axis lesion to be a metastatic paraganglioma from the adrenal mass. We requested a needle

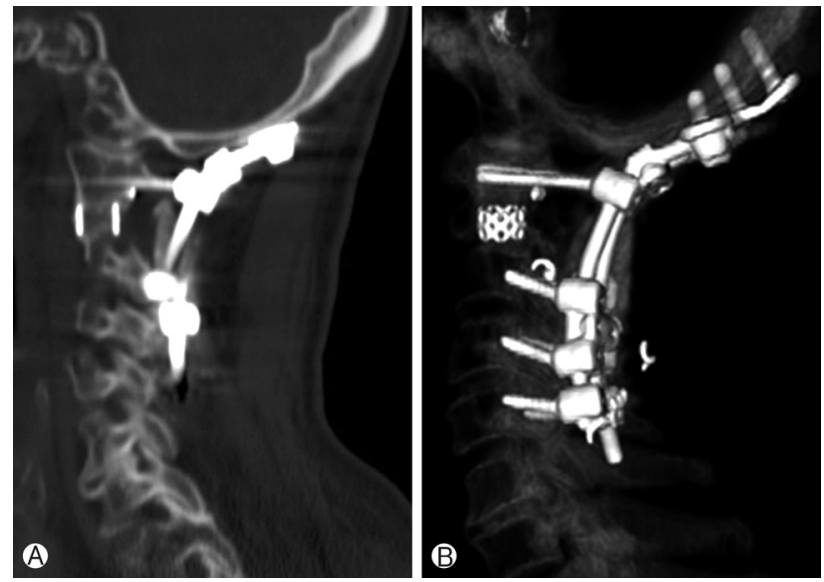

Fig. 4. Postoperative 12 months later, a CT image showing bony bridge formation through the implanted mesh. (A) Sagittal CT image, (B) Postoperative CT 3D reconstruction image.

biopsy from a skeletal radiologist; however, the radiologist recommended an open biopsy and surgery because of the hypervascularity of the tumor and the possibility of neural injury by the needling. On preoperative day 1 , trans-femoral spinal angiography was performed, which revealed that the axis mass was fed from both the vertebral and deep cervical arteries. Feeder embolization was done by embolization particles. Under intra-operative neurophysiological monitoring, removal of the tumor was performed with a Cavitron Ultrasonic Surgical Aspirator (CUSA). After C1 lateral mass screw (posterior arch entry) and lateral mass screw fixation at $\mathrm{C} 3$ to $\mathrm{C} 5$ was applied, the axis tumor's lateral mass and pedicle was removed by CUSA. The tumor compressing spinal cord was grossly removed from the posterior to anterior poles; however, the bony structure around the left $\mathrm{C} 2$ pedicle was severely damaged. Therefore, we reconstructed the atlantoaxial joint with a Harms Cage, a surgical titanium mesh cage, filled with allograft cancellous bone chips. After the occipital plate placement, occipitocervical fixation was performed using a polyaxial screw-rod system with multiple rods (Fig. 4).

The patient's postoperative course was uneventful. A frozen biopsy examination revealed that the tumor tissue was more likely to be metastatic paraganglioma. Nevertheless, the final diagnosis of the tumor was reported as plasmacytoma, following immunohistochemistry (IHC) analysis. The IHC staining of the neoplastic plasma cells revealed immunoglobulin kappa chain and CD138 \& CD56 \& MUM-1 positive expression, and ki-67 positive expression in 3-5\% of cells. Bone marrow aspiration biopsy was performed when the plasmacytoma was diagnosed, and normocellular marrow with a slight increase of plasma cells (3.6\% of nucleated cell count) was observed. Immediate postoperative MRI showed appropriate decompression of the cervical spinal cord. Follow-up CT revealed suc- 
cessful atlantoaxial joint fusion evidenced by bony bridge formation through the implanted mesh. Adjuvant chemotherapy is being administered for myelomatosis, with alkeran and prednisolone to prevent the disease from spreading and to control the remnant enhancing lesion extending to the $\mathrm{C} 4$ ventral epidural space.

\section{DISCUSSION}

Plasmacytoma of the cervical spine is likely to be misdiagnosed as cervical degenerative disease because of the similar clinical features and the sometimes negative findings of plain radiography. In this case, radiography showed expansile, irregular osteolytic lesions, and vertebral instability, without any periosteal reaction. If CT or MRI was conducted in the early period of the disease, the lesions can be detected early.

It is difficult to distinguish plasmacytoma from other osteolytic tumors from radiological results alone. To distinguish it from other diseases, a percutaneous biopsy of the spine is needed, if possible under the guidance of fluoroscopy or CT in consideration of this procedure's risks. A final diagnosis still depends mainly on pathological examination, especially IHC and immunofixation electrophoresis. Monoclonality and/or an aberrant plasma cell phenotype should be demonstrated. Useful markers include CD19, CD56, CD27, CD117, and cyclinD $1^{10,12)}$. In our patient, a left adrenal mass was discovered and diagnosed as either paraganglioma or pheochromocytoma. Therefore, before the operation, we considered the mass to be metastatic paraganglioma. As a result of the frozen biopsy, paraganglioma was suspected, but after the IHC examination, which was performed 7 days after surgery, plasmacytoma was diagnosed.

Although radiotherapy is the treatment of choice for plasmacytoma of the spine, the evidence of its efficacy has been mainly based on small retrospective studies ${ }^{1,3,5-9,11,13,14)}$. Some patients suffer from tumor invasion into the spinal canal, causing extremity dysfunction or paraplegia as a result of spinal cord or nerve root compression. In these conditions, most spine surgeons believe that radiotherapy cannot be a substitute for surgery. Although surgery (partial or complete resection and radiotherapy versus radiotherapy alone) did not influence the 10-year probability of local control, it aims at relieving spinal cord and nerve root compression through excision of the tumor and reconstruction of spinal stability. We believe that the potential surgical outcome frequently outweighs the risk of surgery. In this case, the axis mass was safely removed without spinal cord or cervical root injury and with adequate stabilization using a polyaxial screw-rod system with multiple rods.

The general prognosis of SBP is comparatively better than plasmacytoma, with a 5 -year survival rate of about $70 \%$ and median overall survival period of 7.5 to 12 years ${ }^{2,3}$. Patients, especially those with plasmacytoma of the spine, will probably eventually develop multiple myeloma (MM) in the future, with a median delay of 2 to 4 years. There is no effective method to prevent plasmacytoma from progressing to $\mathrm{MM}$, and there is no consensus in the literature about these adverse prognostic features.

\section{CONCLUSION}

We presented a rare case of plasmacytoma of the axis, mimicking metastatic paraganglioma, and treated by a posterior decompressive surgery with reconstruction using a polyaxial screw-multiple rod system with a Hams cage.

\section{REFERENCES}

1. Chak LY, Cox RS, Bostwick DG, Hoppe RT: Solitary plasmacytoma of bone: treatment, progression, and survival. J ClinOncol 5:1811-1815, 1987

2. Dimopoulos MA, Hamilos G: Solitary bone plasmacytoma and extramedullary plasmacytoma. Curr Treat Options Oncol 3:255259, 2002

3. Dimopoulos MA, Moulopoulos LA, Maniatis A, Alexanian R: Solitary plasmacytoma of bone and asymptomatic multiple myeloma. Blood 96:2037-2044, 2000

4. Grogan TM, Muller-Hermelink HK, Van CB, Harris NL, Kyle RA: Plasm cell neoplasms in Jaffe ES, Harris NL, Stein H, Vardiman JW(eds): World health organization classification of tumours. Pathology and genetics of tumours of haematopoietic and lymphoid tissues. Lyon: IARC Press, pp142-156, 2001

5. Ghogawala Z, Mansfield FL, Borges LF: Spinal radiation before surgical decompression adversely affects outcomes of surgery for symptomatic metastatic spinal cord compression. Spine 26: 818-821, 2001

6. Hu K, Yahalom J: Radiotherapy in the management of plasma cell tumors. Oncology 14:101-108, 2000

7. Jyothirmayi R, Gangadharan VP, Nair MK, Rajan B: Radiotherapy in the treatment of solitary plasmacytoma. $\mathrm{Br} \mathrm{J}$ Radiol 70: 511-516, 1997

8. Liebross RH, Ha CS, Cox JD, Weber D, Delasalle K, Alexanian R: Solitary bone plasmacytoma: outcome and prognostic factors following radiotherapy. Int J Radiat Oncol Biol Phys 41:10631067, 1998

9. Mendenhall CM, Thar TL, Million RR: Solitary plasmacytoma of bone and soft tissue. Int J Radiat Oncol Biol Phys 6:14971501, 1980

10. Ozsahin M, Tsang RW, Poortmans P, Belkacémi Y, Bolla M, Dinçbas FO, et al: Outcomes and patterns of failure in solitary plasmacytoma: a multicenter Rare Cancer Network study of 258 patients. Int J Radiat Oncol Biol Phys 64:210-217, 2006

11. Rawstron AC, Orfao A, Beksac M, Bezdickova L, Brooimans 
RA, Bumbea H, et al: Report of the European Myeloma Network on multiparametric flow cytometry in multiple myeloma and related disorders. Haematologica 93:431-438, 2008

12. Soutar R, Lucraft H, Jackson G: Guidelines on the diagnosis and management of solitary plasmacytoma of bone and solitary extramedullary plasmacytoma. Clin Oncol 16:405-413, 2004

13. Stephens EA, Boll M, O’Connor S, Rawstron AC, Jack AS, Owen RG: Soft tissue plasmacytomas are characterised by a "myeloma-like" immunophenotype and genotype and low level bone marrow involvement. Br J Haematol 129(Supp 1):24-25, 2005

14. Tsang RW, Gospodarowicz MK, Pintilie M, Bezjak A, Wells W, Hodgson DC, et al: Solitary plasmacytoma treated with radiotherapy: impact of tumor size on outcome. Int J Radiat Oncol Biol Phys 50:113-120, 2001 